\title{
Surgically resected human tumors reveal the biological significance of the gastric cancer stem cell markers CD44 and CD26
}

\author{
SHIMPEI NISHIKAWA ${ }^{1,2}$, MASAMITSU KONNO ${ }^{1}$, ATSUSHI HAMABE ${ }^{1,2}$, SHINICHIRO HASEGAWA $^{1,2}$, \\ YOSHIHIRO KANO ${ }^{1,2}$, TAKAHITO FUKUSUMI ${ }^{3}$, TAROH SATOH ${ }^{1}$, SHUJI TAKIGUCHI ${ }^{2}$, \\ MASAKI MORI $^{2}$, YUICHIRO DOKI ${ }^{2}$ and HIDESHI ISHII ${ }^{1}$ \\ Departments of ${ }^{1}$ Frontier Science for Cancer and Chemotherapy, ${ }^{2}$ Gastroenterological Surgery \\ and ${ }^{3}$ Otorhinolaryngology-Head and Neck Surgery, Graduate School of Medicine, \\ Osaka University, Suita, Osaka 565-0871, Japan
}

Received March 9, 2014; Accepted August 29, 2014

DOI: $10.3892 / \mathrm{ol} .2015 .3063$

\begin{abstract}
Cancer tissue is maintained by relatively small populations of cancer stem cells (CSCs), which are involved in chemotherapy resistance, recurrence and metastasis. As tumor tissues are comprised of various cells, studies of human clinical samples are important for the characterization of CSCs. In the present study, an expression profiling study was performed in which an anti-cell surface marker antibody-based array platform, a flow cytometry-based cell separation technique and a tumorigenicity analysis in immunodeficient animals were utilized. These approaches revealed that the markers cluster of differentiation (CD)44 and CD26 facilitated the fractionation of surgically resected human gastric cancer (GC) cells into the following subset populations with distinct tumorigenic potentials: Highly tumorigenic $\mathrm{CD} 26^{+} \mathrm{CD} 44^{+}$cells $(6 / 6$ mice formed tumors), moderately tumorigenic CD26+CD44- cells (5/6 mice formed tumors), and weakly or non-tumorigenic CD26-CD44 cells (2/6 mice formed tumors). Furthermore, exposure to 5-fluorouracil significantly increased the proportion of $\mathrm{CD} 26^{+}$cells in vitro. The present study demonstrated that the combined expression of CD26 and CD44 presents a potential marker of human GC stem cells.
\end{abstract}

Correspondence to: Dr Masaki Mori, Department of Gastrointestinal Surgery, Graduate School of Medicine, Osaka University, 2-2 (E2) Yamadaoka, Suita, Osaka 565-0871, Japan E-mail:mmori@gesurg.med.osaka-u.ac.jp

Dr Hideshi Ishii, Department of Frontier Science for Cancer and Chemotherapy, Graduate School of Medicine, Osaka University, 2-2 (E2) Yamadaoka, Suita, Osaka 565-0871, Japan

E-mail: hishii@gesurg.med.osaka-u.ac.jp

Key words: gastric cancer, cancer stem cell, tumor-initiating cell, chemotherapy, CD44, CD26

\section{Introduction}

More than 800,000 mortalities occur annually as a result of gastric cancer (GC), which is the second leading cause of cancer-associated mortality worldwide (1). Although the surgical techniques, chemoradiation therapy regimens, and pre- and postoperative medications used to treat GC have improved, the median overall survival time of patients with advanced GC remains at $\sim 13$ months (2). Tumor heterogeneity has been identified as a factor that negatively affects patient survival and numerous studies have indicated that the involvement of small populations of cancer stem cells (CSCs) within tumor tissues may contribute to this heterogeneity and thus to chemoradiation therapy resistance, local invasion and metastasis to other organs (3-14).

The CSC hypothesis was initially proposed following the prospective identification of leukemia-initiating cells in patients with acute myeloid leukemia (AML) $(3,4)$. The AML studies, which were conducted in immunocompromised mice, revealed a significantly higher leukemic potential in cluster of differentiation (CD) $34^{+} \mathrm{CD} 38^{-}$leukemic cells than in $\mathrm{CD} 34^{+} \mathrm{CD} 38^{+}$or $\mathrm{CD} 34^{-}$cells $(3,4)$. Following the identification of these leukemic stem cells, CSCs or cancer-initiating cells have been identified in various types of solid tumors, including breast, brain, colon, pancreatic, liver and gastric tumors (5-11). Xenotransplantation assays have demonstrated the high capacity of CSCs for self-renewal and, thereby, the likelihood that these cells may regenerate the heterogeneous types of cancer cells that complicate therapeutic approaches $(12,13)$. When characterizing CSCs, the use of human samples, which have usually been resected via surgical procedures, is important to elucidate the mechanisms of chemoresistance, radioresistance, metastasis and recurrence, and to identify novel drug targets against neoplastic diseases with poor prognoses $(12,14)$.

Regarding gastric CSC surface markers, several molecules or combinations of molecules have been reported to indicate cell subsets, including $\mathrm{CD} 44^{+}, \mathrm{CD} 44^{+} \mathrm{EpCAM}^{+}, \mathrm{CD} 44^{+} \mathrm{CD} 24^{+}$, and $\mathrm{CD} 90^{+}$cells, as well as cells with high aldehyde dehy- 

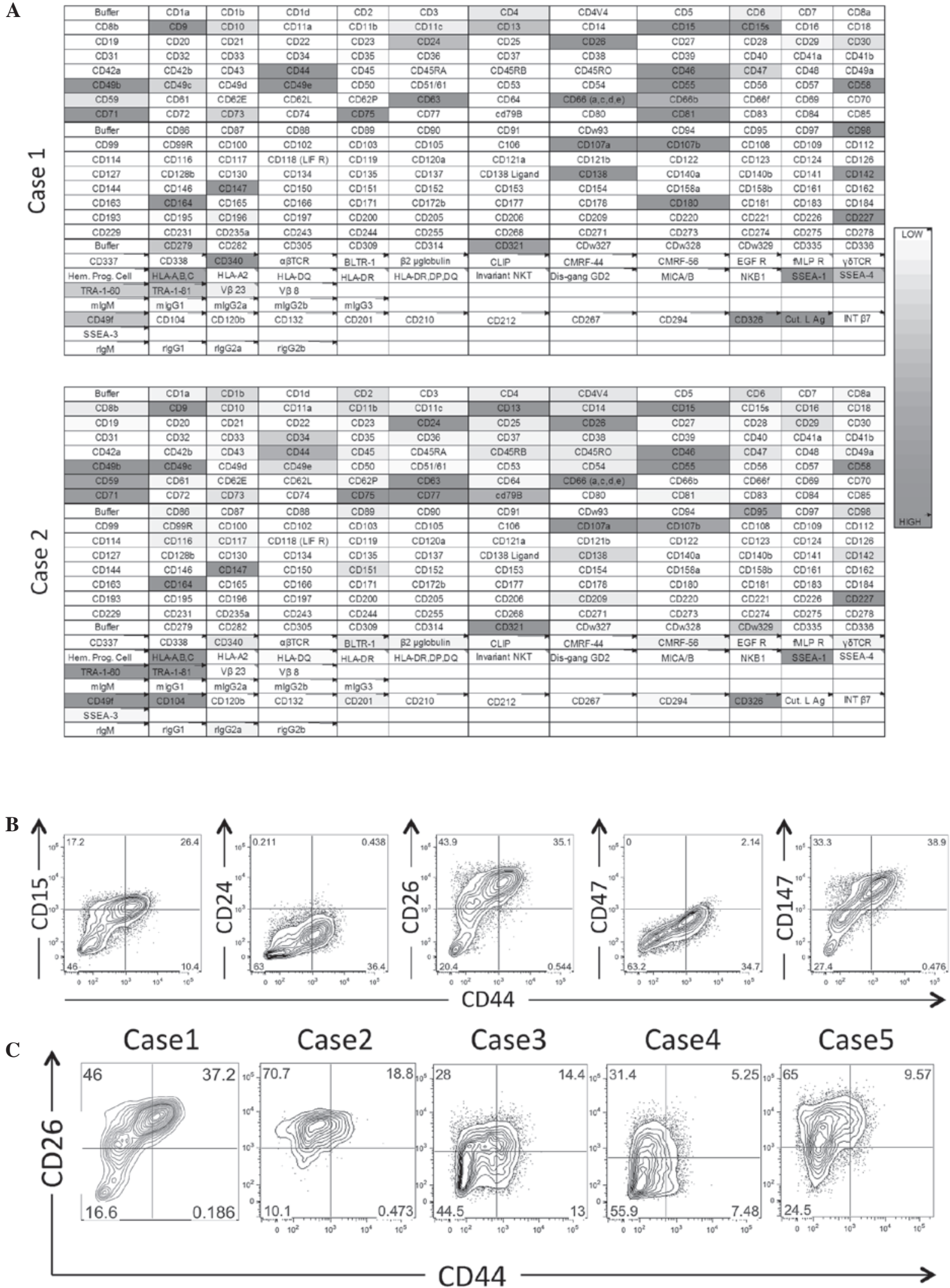

Figure 1. Surface marker screening of surgical specimen-derived xenografts. (A) Heat map of surface marker screening. (B) Multicolor flow cytometric analysis of candidate surface markers. (C) Five representative cases of flow cytometric analyses using CD26 and CD44. CD, cluster of differentiation.

drogenase activity $(11,15-20)$. However, the hyaluronic acid receptor, CD44, cannot be used to specifically detect gastric CSCs, even when combined with CD133 (21). In the present study, the cell surface markers expressed on surgically resected GC cells were comprehensively profiled in combination with a xenograft study in immunodeficient mice to identify novel 
A

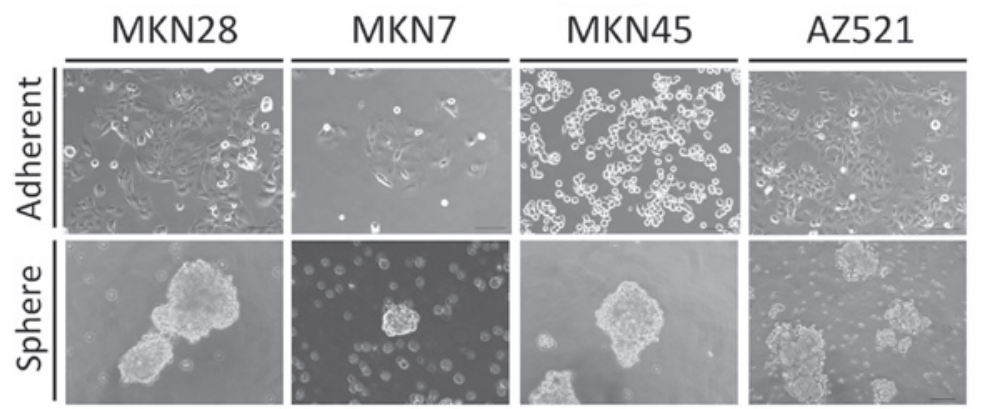

B

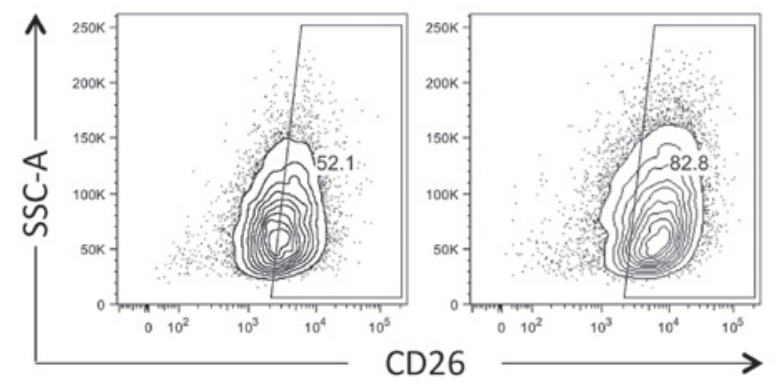

C

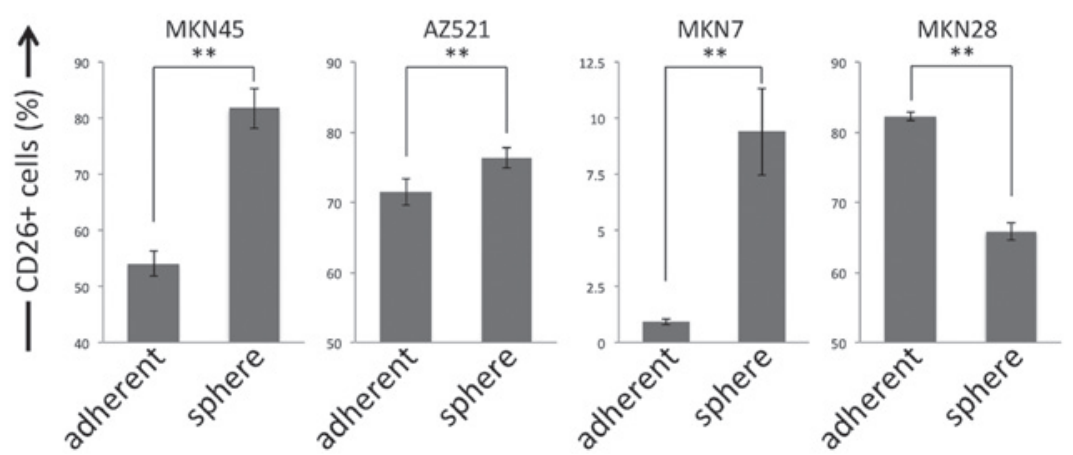

Figure 2. Sphere formation of CD26/CD44 expression-defined subsets.(A) GC cell line morphologies in adherent or spherical states. (B) Representative increased CD26 expression data following sphere formation. (C) CD26 expression following sphere formation in four GC cell lines. CD, cluster of differentiation; GC, gastric cancer.

markers for the detection of gastric CSCs. This approach facilitated the identification of the combination of CD44 and CD26 as a useful marker of cancer stem cell properties.

\section{Materials and methods}

Cancer cells. Fresh surgically resected well-differentiated GC specimens were obtained at Osaka University Medical Hospital (Osaka, Japan) between 2011 and 2012 and written informed consent was obtained from all patients. This study was approved by the ethics committee of Osaka University (Osaka, Japan). The GC cell lines (MKN7, MKN28, MKN45 and AZ521, were provided by the Department of Gastroenterological Surgery, Osaka University) were cultured in RPMI-1640 medium (Sigma-Aldrich, St. Louis, MO, USA) supplemented with $10 \%$ fetal bovine serum (FBS; Thermo Fisher Scientific Inc., Waltham, MA, USA) and an antibiotic/antimycotic solution (Sigma-Aldrich). To facilitate sphere formation, the cells were cultured in Dulbecco's modified Eagle's medium (DMEM)/F12 medium supplemented with $20 \mathrm{ng} / \mathrm{ml}$ of recombinant human epidermal growth factor (EGF; Promega Corporation, Madison, WI, USA) and $10 \mathrm{ng} / \mathrm{ml}$ of basic fibroblast growth factor (FGF; PeproTech
Inc., Rocky Hill, NJ, USA) in 96-well plates, six-well plates, or 100-mm dishes with Ultra-Low Attachment surfaces (Corning Inc., Corning, NY, USA).

Antibody arrays and flow cytometric studies. A Lyoplate ${ }^{\circledR}$ assay (BD Biosciences, San Jose, CA, USA) was used to screen surface markers, and was equipped with antibodies against 242 human cell surface markers, (mouse anti-human monoclonal antibodies: CD1a, CD1b, CD1d, CD2, CD3, CD4, CD4v4, CD5, CD6, CD7, CD8a, CD8b, CD9, CD10, CD11a, CD11b, CD11c, CD13, CD14, CD15, CD15s, CD16, CD18, CD19, CD20, CD21, CD22, CD23, CD24, CD25, CD26, CD27, CD28, CD29, CD 30, CD31, CD 32, CD 33, CD 34, CD 35, CD36, CD37, CD38, CD39, CD40, CD41a, CD41b, CD42a, CD42b, CD43, CD44, CD45, CD45RA, CD45RB, CD45RO, CD46, CD47, CD48, CD49a, CD49b, CD49c, CD49d, CD49e, CD50, CD51/61, CD53, CD54, CD55, CD56, CD57, CD58, CD59, CD61, CD62E, CD62L, CD62P, CD63, CD64, CD66a, -c, -d, -e), CD66b, CD66f, CD69, CD70, CD71, CD72, CD73, CD74, CD75, CD77, CD79b, CD80, CD81, CD83, CD84, CD85, CD86, CD87, CD88, CD89, CD90, CD91, CDw93, CD94, CD95, CD97, CD98, CD99, CD99R, CD100, CD102, CD103, CD105, CD106, CD107a, CD107b, CD108, CD109, CD112, 
A

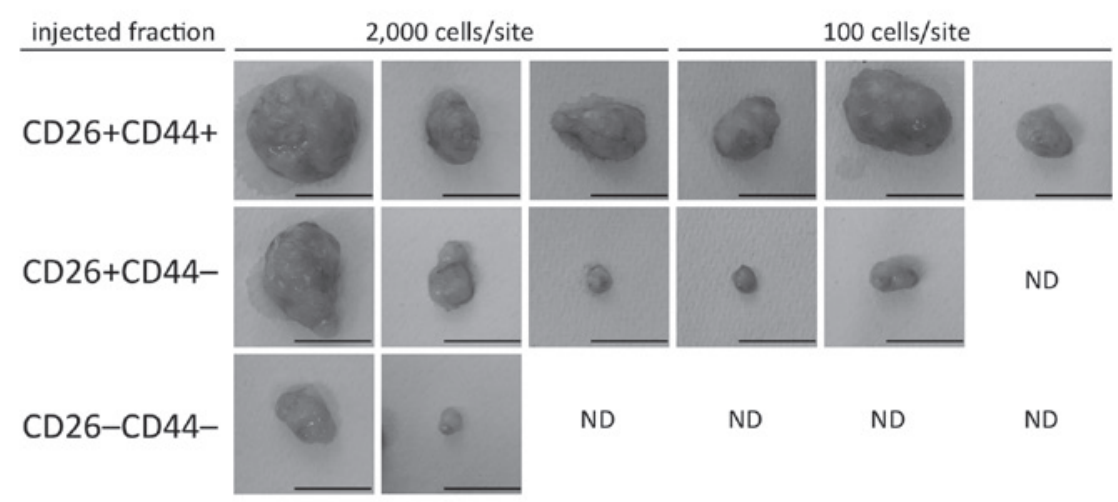

B

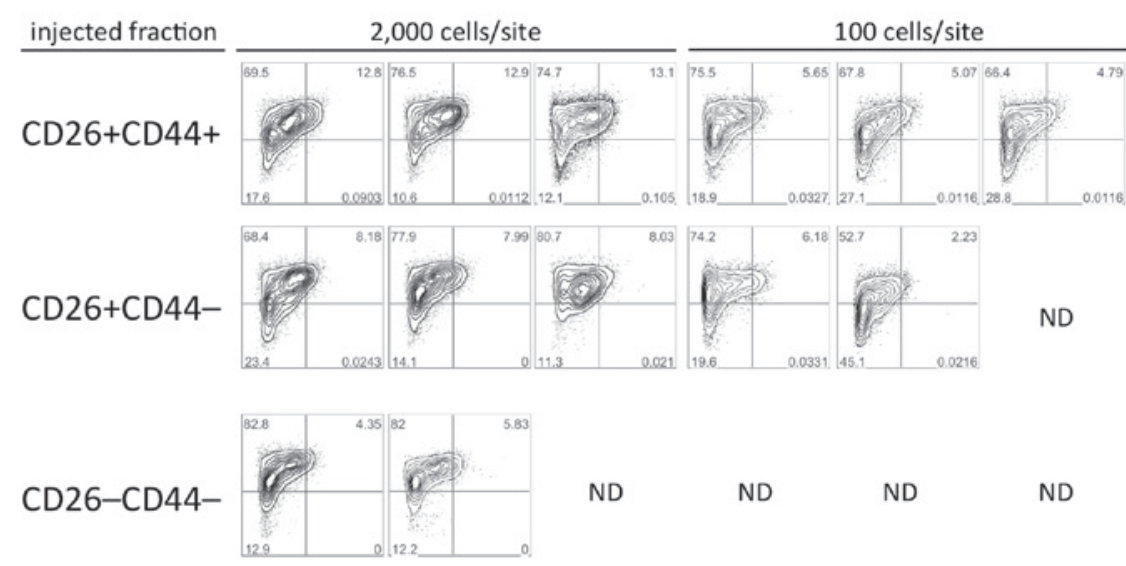

C

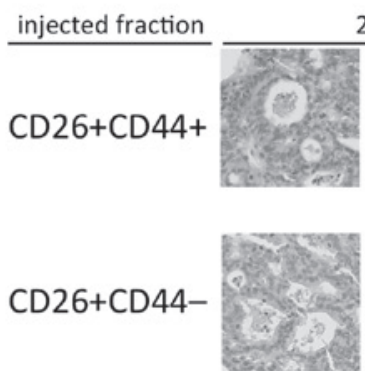

2,000 cells/site

100 cells/site
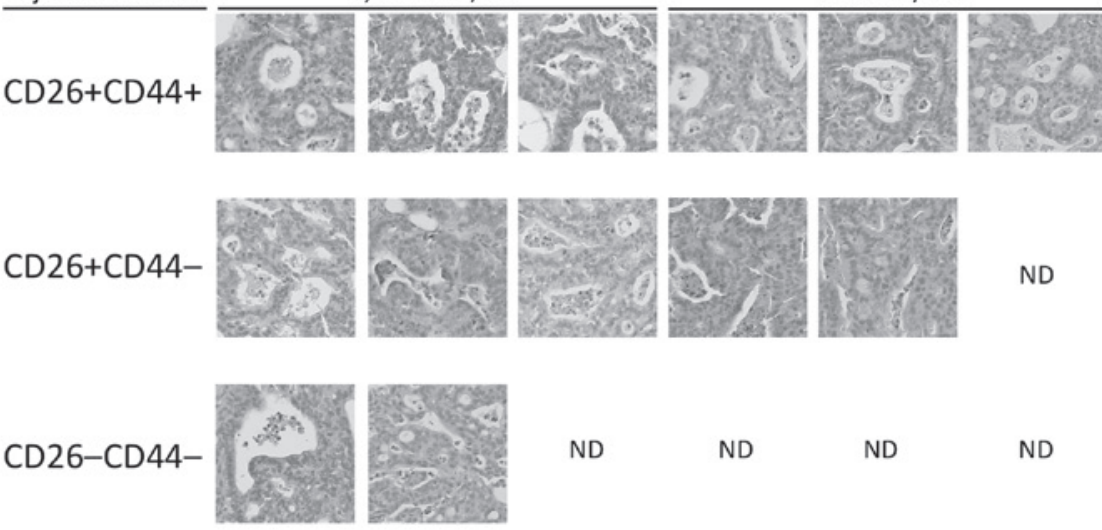

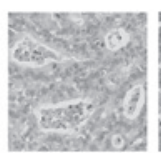

ND

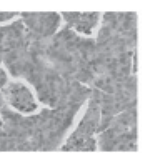

ND

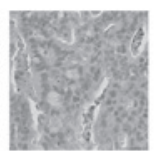

ND

ND
ND

Figure 3. Tumorigenicity of CD26/CD44 expression-defined subsets. (A) Excised tumors formed following the injection of cell subsets. (B) Flow cytometric tumor re-analysis. (C) Histological tumor analysis (hematoxylin-eosin staining). CD, cluster of differentiation.

CD114, CD116, CD117, CD118, CD119, CD120a, CD121a, CD121b, CD122, CD123, CD124, CD126, CD127, CD128b, CD130, CD134, CD135, CD137, CD137 ligand, CD138, CD140a, CD140b, CD141, CD142, CD144, CD146, CD147, CD150, CD151, CD152, CD153, CD154, CD158a, CD158b, CD161, CD162, CD163, CD164, CD165, CD166, CD171, CD279, CD282, CD305, CD309, CD314, CD321, CDw327, CDw328, CDw329, CD335, CD336, CD337, CD338, CD304, $\alpha \beta T$ CR, $\beta 2$-microglobulin, human leukotriene B4 receptor-1, CLIP, CMRF-44, CMRF-56, epidermal growth factor receptor, fMLP receptor, $\gamma \delta T C R$, hybrid protein complex, human leukocyte antigen (HLA)-A, -B, -C, HLA-A2, HLA- DQ, HLA- DR, HLA-DR, -DP, -DQ, Invariant NK T, Disialoganglioside GD2,
MIC A/B, NKB1, stage-specific embryonic antigen (SSEA)-1, SSEA- 4, TRA-1-60, TRA-1-81, V $\beta$ 23, V $\beta 8$ and CD326; rat anti-human monoclonal antibodies: CD49f, CD104, CD120b, CD132, CD201, CD210, CD212, CD267, CD294, SSEA4, cutaneous lymph antigen and Integrin $\beta 7$ ), according to the manufacturer's instructions. Briefly, to evaluate the cell lines, the cells were detached from the culture dishes (Iwaki \& Co., Ltd. Tokyo, Japan) using the Accutase ${ }^{\mathrm{TM}}$ Cell Detachment Solution (BD Biosciences) and were washed with Dulbecco's phosphate-buffered saline (Wako Pure Chemical Industries, Ltd., Osaka, Japan). To evaluate fresh surgical specimens or xenografts, the tumor tissues were dissected into small sections, and digested with collagenase H (Roche Diagnostics, 
Basel, Switzerland) and DNase I (Worthington Biochemical Corporation, Lakewood, NJ, USA) for $1 \mathrm{~h}$ at $37^{\circ} \mathrm{C}$. Next, the digested tissues were filtered through a $100-\mu \mathrm{m}$ filter, layered over Ficoll 1077 (GE Healthcare Life Sciences, Pittsburgh, PA, USA) and centrifuged to remove dead cells and debris. The resuspended cells were treated with TruStain fc $\mathrm{X}^{\mathrm{TM}}$ monoclonal rat anti-mouse CD16/32 antibody (BioLegend, Inc., San Diego, CA, USA) and a human FcR blocking reagent (Miltenyi Biotec GmbH, Bergisch Gladbach, Germany) to block the Fc receptors, followed by staining with antibodies against CD26-phycoerythrin (PE), CD24, CD44, CD47 and CD147 (BD Biosciences); CD26-PE-Cyanin 5 (BioLegend); and CD15 (Beckman-Coulter, Indianapolis, IN, USA) for $30 \mathrm{~min}$ to evaluate the surface expression levels. To exclude mouse cells, the cells were first incubated with a biotinylated anti-mouse Lineage Panel (monoclonal rat TER-119, CD11b, Gr-1, CD45R/B220 and hamster CD3e antibodies), monoclonal rat anti-mouse $\mathrm{H}-2 \mathrm{Kd}$ and CD31 antibodies (all purchased from BioLegend), followed by streptavidin-conjugated Pacific Blue (Invitrogen Life Technologies, Carlsbad, CA, USA). Human GC cell lines were cultured in RPMI-1640 medium supplemented with $10 \%$ FBS with or without 5FU. For sphere formation, the cell lines were cultured for $7 \mathrm{~d}$ in Ultra-low Attachment dishes (Corning Inc.) at a density of $\leq 5 \times 10^{6}$ cells/dish. Briefly, the cells were collected and washed to remove serum and then suspended in serum-free DMEM/F12 supplemented with $20 \mathrm{ng} / \mathrm{ml}$ of human recombinant EGF, $10 \mathrm{ng} / \mathrm{ml}$ human recombinant basic FGF, 2\% B27 supplement without vitamin A and 1\% N2 supplement (Invitrogen Life Technologies). The spheres with a diameter of $>50 \mu \mathrm{m}$ were counted and collected via gentle centrifugation with a pipette.

Tumorigenicity. A total of 24 four- to six-week-old female non-obese diabetic/severe combined immunodeficiency (NOD/SCID) mice (CLEA Japan Inc., Tokyo, Japan) were used as subcutaneous xenograft models under specific pathogen-free conditions. The tumor volumes were calculated using the following formula: Tumor volume $=$ (longest diameter) $\mathrm{x}$ (shortest diameter $)^{2} \mathrm{x} 0.5$. This study was approved by the Animal Experiments Committee of Osaka University.

Statistical analysis. Data are expressed as the mean \pm standard deviation. Continuous variables were compared using the Student's t-test. Statistical analyses were performed using JMP statistical software, version 9.0 (SAS Institute Inc., Cary, NC, USA).

\section{Results}

Antibody-based array and flow cytometry. Two surgically resected GC xenografts were assessed for surface marker expression via flow cytometry (Fig. 1A). The antibody-based array study indicated that 27 of the 242 evaluated surface markers were expressed in the two xenografts. The subsequent multicolor flow cytometric analysis confirmed that among these markers, CD15, CD24, CD26, CD44, CD47 and CD147 extended the expression profiling from the negative to the positive range and further revealed that the combination of CD26 and CD44 may be used to fractionate the GC xenograft cells
A

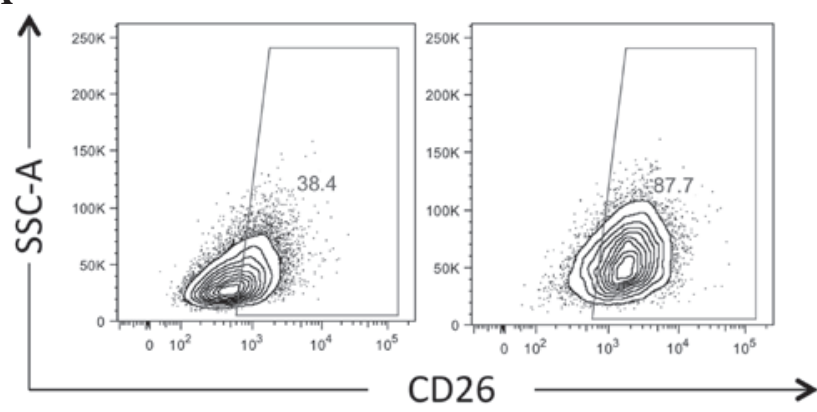

B

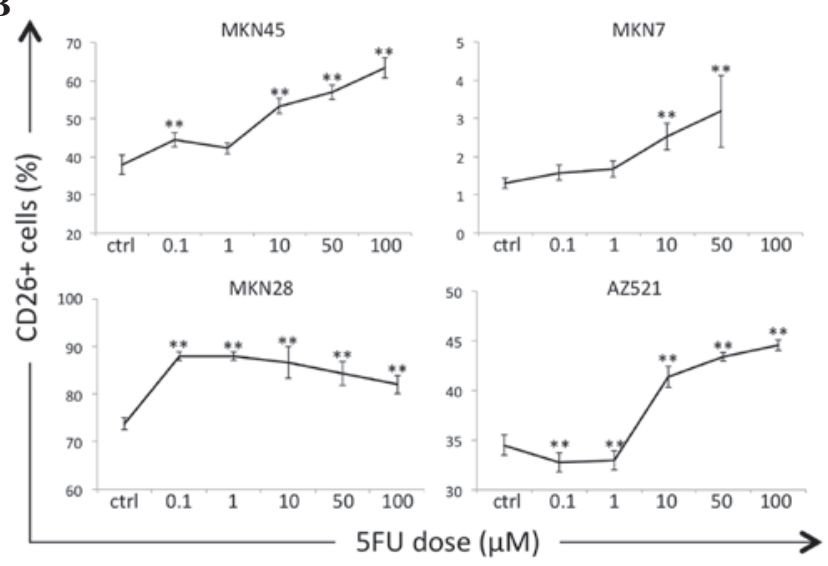

C

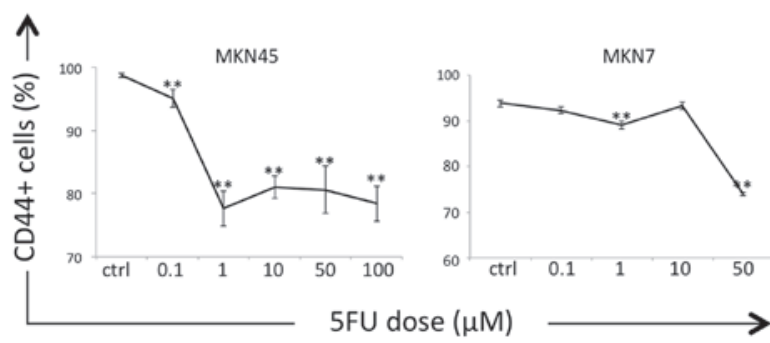

Figure 4. Chemoresistant subsets according to CD26/CD44 expression. (A) Representative flow cytometric data following exposure to chemotherapeutic agents. Percentages of (B) $\mathrm{CD} 26^{+}$and (C) $\mathrm{CD} 44^{+}$cells following exposure to $5 \mathrm{FU}$. CD, cluster of differentiation; 5FU, 5 -fluorouracil.

into three subsets (Fig. 1B). The confirmation study indicated that five of the flow cytometrically analyzed cases were consistently fractionated into at least three subsets according to the combined CD26 and CD44 expression statuses (Fig. 1C).

Sphere formation induces $C D 26^{+}$cells. Cell culture conditions suitable for sphere formation have been reported to support stem-like cell maintenance and, presumably, to induce at least partial CSC induction, which consequently enriches CSC cell populations $(6,22)$. Four GC cell lines (MKN7, MKN28, MKN45 and AZ521) were used in a sphere formation assay. Distinct spheres were formed after culturing the cell lines in serum-free and unattached conditions (Fig. 2A). Flow cytometric analysis of the adherent cells and spheres revealed that sphere formation enriched the $\mathrm{CD} 26^{+}$cell subset in three out of four of the examined cell lines (Fig. 2B and C).

$C D 26^{+} C D 44^{+}$cells exhibit high tumorigenic potential. The tumorigenicity of each CD26- and CD44-defined subset was 
assessed. Cells from a surgically obtained specimen-derived GC xenograft (Case 1) were sorted via fluorescence-activated cell sorting into three subsets, $\mathrm{CD} 26^{+} \mathrm{CD} 44^{+}, \mathrm{CD} 26^{+} \mathrm{CD} 44^{-}$, and $\mathrm{CD}^{2} 6^{-} \mathrm{CD}_{4}^{-}$, and were then subcutaneously injected into NOD/SCID mice. The largest and most frequent tumor formations were obtained with the $\mathrm{CD} 26^{+} \mathrm{CD} 44^{+}$subset. The subset formed relatively small tumors; however, the frequency of tumor formation was higher with $\mathrm{CD} 26^{+} \mathrm{CD} 44^{-}$subset than with the CD26-CD44 subset (Fig. 3A). Flow cytometric analysis of the formed tumors revealed the recapitulation of three subsets similar to those in the original xenograft (Figs. 1C and $3 \mathrm{~B}$ ). The histological characteristics of the formed tumors were similar to those of the xenograft prior to sorting and of the original patient sample (Fig. 3C).

Roles of CD26 and CD44 in chemoresistance. Chemoresistance is a clinically important feature of CSCs. GC cells cultured in the presence of 5FU exhibited increased frequencies (\%) of $\mathrm{CD} 26^{+}$cells in a 5FU dose-dependent manner (Fig. 4A and B). In contrast to $\mathrm{CD} 26$, the frequencies of $\mathrm{CD} 44^{+}$cells decreased following 5FU exposure (Fig. 4C). The MKN28 and AZ521 cells were completely CD44- prior to and following 5FU exposure, as determined via flow cytometry (data not shown).

\section{Discussion}

Monoclonal antibodies have been used to identify and characterize cell surface molecules, and similar techniques have been utilized in various research fields, including immunology, hematopoietic stem cell biology and cancer stem cell biology (23). To date, $>350$ cell surface molecules are numbered according to $\mathrm{CD}$ nomenclature; however, a recent bioinformatics study reported that 3,702 transmembrane proteins are expressed on the surfaces of human cells (24).

To the best of our knowledge, there have been no comprehensive reports of cell surface molecule expression evaluations in surgically resected primary GC using $>242$ molecules, as evaluated in the current study. Among the screened surface markers in this study, the combination of CD26 and CD44 was shown to be capable of dividing GC xenograft cells into three to four subsets via flow cytometry (Fig. 1B and C). In a xenotransplantation experiment, similar tumorigenic efficiencies were demonstrated in the $\mathrm{CD} 26^{+} \mathrm{CD} 44^{+}$and $\mathrm{CD} 26^{+} \mathrm{CD} 44^{-}$cells, in contrast to the low tumorigenicity of the CD26-CD44- subset (Fig. 3A and B). These data indicate that the insufficiency of CD44 as a CSC marker may be improved by the complementary use of CD26 as a co-marker.

As a result of the CSC hypothesis, cancer cells within whole tumor tissues have been classified into two major groups, the tumorigenic and non-tumorigenic CSCs (non-CSCs). However, recently, tumorigenic cell heterogeneity with respect to clonal dominancy and chemoresistance was identified using of clonal tracking techniques based on lentiviral insertion sites $(25,26)$. Kreso et al (25) reported five types of clones within colorectal cancer xenografts. In contrast to actively proliferating clones, the slow-growing cell types supposedly became dominant following chemotherapy (25). In the present study, CD $26{ }^{+} \mathrm{CD} 44^{+}$ cells formed relatively larger tumors when compared with CD26+CD44- cells (Fig. 3A). This observation indicates that $\mathrm{CD} 26^{+} \mathrm{CD} 44^{+}$cells underwent rapid proliferation, unlike the
CD $26^{+} \mathrm{CD} 44^{-}$cells. However, following 5FU exposure, the frequencies of $\mathrm{CD} 44^{+}$cells decreased significantly in vitro, whereas the frequencies of $\mathrm{CD}_{2} 6^{+}$cells increased (Fig. 4). These results indicate that although $\mathrm{CD} 26^{+} \mathrm{CD} 44^{+}$cells and $\mathrm{CD} 26^{+} \mathrm{CD} 44^{-}$cells may initiate tumor growth and regenerate histologically and phenotypically similar tumors (Fig. 3B and C), the cell subsets may differ with respect to proliferative activity and chemotherapeutic sensitivity. Consequently, the role of CSC in therapeutic resistance remains clinically important. The identification of a therapy-resistant CSC subset may accelerate the process of CSC hypothesis-based novel therapy development.

\section{Acknowledgements}

The authors would like to thank Miyuki Ozaki and Yuko Noguchi for their technical support. The current study was partly supported by a Core Research Grant-in-Aid for Scientific Research from the Ministry of Education, Culture, Sports, Science and Technology in Japan (to M.K., H.I. and M.M.; grant nos. ; 26670604, 23390199 and 21229015); a Grant-in-Aid from the Third Term Comprehensive 10-year Strategy for Cancer Control of the Ministry of Health, Labor, and Welfare in Japan (to H.I. and M.M.; grant no. H26-018); a grant from the Kobayashi Cancer Research Foundation (to H.I.; grant no. H24); a grant from the Princess Takamatsu Cancer Research Fund, Japan (to H.I.; grant no. H22); and a grant from the SENSHIN Medical Research Foundation (to H.I.; grant no. H25).

The authors received partial support from Chugai Pharmaceutical Co., Ltd. (Tokyo, Japan), Yakult Honsha Co., Ltd. (Tokyo, Japan), and Takeda Pharmaceutical Co., Ltd (Osaka, Japan).

\section{References}

1. Ferlay J, Shin HR, Bray F, Forman D, Mathers C and Parkin DM: Estimates of worldwide burden of cancer in 2008: GLOBOCAN 2008. Int J Cancer 127: 2893-2917, 2010.

2. Bang YJ, Van Cutsem E, Feyereislova A, et al; ToGA Trial Investigators: Trastuzumab in combination with chemotherapy versus chemotherapy alone for treatment of HER2-positive advanced gastric or gastro-oesophageal junction cancer (ToGA): a phase 3, open-label, randomised controlled trial. Lancet 376: 687-697, 2010.

3. Lapidot T, Sirard C, Vormoor J, et al: A cell initiating human acute myeloid leukemia after transplantation into SCID mice. Nature 367: 645-648, 1994.

4. Bonnet D and Dick JE: Human acute myeloid leukemia is organized as a hierarchy that originates from a primitive hematopoietic cell. Nat Med 3: 730-737, 1997.

5. Al-Hajj M, Wicha MS, Benito-Hernandez A, Morrison SJ and Clarke MF: Prospective identification of tumorigenic breast cancer cells. Proc Natl Acad Sci USA 100: 3983-3988, 2003.

6. Singh SK, Hawkins C, Clarke ID, et al: Identification of human brain tumor initiating cells. Nature 432: 396-401, 2004.

7. O'Brien CA, Pollett A, Gallinger S and Dick JE: A human colon cancer cell capable of initiating tumor growth in immunodeficient mice. Nature 445: 106-110, 2007.

8. Ricci-Vitiani L, Lombardi DG, Pilozzi E, et al: Identification and expansion of human colon-cancer-initiating cells. Nature 445: 111-115, 2007.

9. Li C, Heidt DG, Dalerba P, et al: Identification of pancreatic cancer stem cells. Cancer Res 67: 1030-1037, 2007.

10. Haraguchi $\mathrm{N}$, Ishii $\mathrm{H}$, Mimori $\mathrm{K}$, et al: $\mathrm{CD} 13$ is a therapeutic target in human liver cancer stem cells. J Clin Invest 120: 3326-3339, 2010.

11. Takaishi S, Okumura T, Tu S, et al: Identification of gastric cancer stem cells using the cell surface marker CD44. Stem Cells 27: 1006-1020, 2009 
12. Clarke MF, Dick JE, Dirks PB, et al: Cancer stem cells - perspectives on current status and future directions: AACR Workshop on cancer stem cells. Cancer Res 66: 9339-9344, 2006.

13. O'Brien CA, Kreso A and Jamieson CH: Cancer stem cells and self-renewal. Clin Cancer Res 16: 3113-3120, 2010.

14. Medema JP: Cancer stem cells: the challenges ahead. Nat Cell Biol 15: 338-344, 2013.

15. Fukuda K, Saikawa Y, Ohashi M, et al: Tumor initiating potential of side population cells in human gastric cancer. Int J Oncol 34 1201-1207, 2009.

16. Han ME, Jeon TY, Hwang SH, et al: Cancer spheres from gastric cancer patients provide an ideal model system for cancer stem cell research. Cell Mol Life Sci 68: 3589-3605, 2011.

17. Zhang C, Li C, He F, Cai Y and Yang H: Identification of $\mathrm{CD} 44^{+} \mathrm{CD} 24^{+}$gastric cancer stem cells. J Cancer Res Clin Oncol 137: 1679-1686, 2011.

18. Jiang J, Zhang Y, Chuai S, et al: Trastuzumab (herceptin) targets gastric cancer stem cells characterized by CD90 phenotype. Oncogene 31: 671-682, 2012.

19. Katsuno Y, Ehata S, Yashiro M, Yanagihara K, Hirakawa K and Miyazono K: Coordinated expression of REG4 and aldehyde dehydrogenase 1 regulating tumourigenic capacity of diffuse-type gastric carcinoma-initiating cells is inhibited by TGF- $\beta$. J Pathol 228: 391-404, 2012.
20. Nishikawa S, Konno M, Hamabe A, et al: Aldehyde dehydrogenase high gastric cancer stem cells are resistant to chemotherapy. Int J Oncol 42: 1437-1442, 2013.

21. Rocco A, Liguori E, Pirozzi G, et al: CD133 and CD44 cell surface markers do not identify cancer stem cells in primary human gastric tumors. J Cell Physiol 227: 2686-2693, 2012.

22. Ponti D, Costa A, Zaffaroni N, et al: Isolation and in vitro propagation of tumorigenic breast cancer cells with stem/progenitor cell properties. Cancer Res 65: 5506-5511, 2005.

23. Zola H: Medical applications of leukocyte surface molecules - the CD molecules. Mol Med 12: 312-316, 2006.

24. da Cunha JP, Galante PA, de Souza JE, et al: Bioinformatics construction of the human cell surfaceome. Proc Natl Acad Sci USA 106: 16752-16757, 2009.

25. Kreso A, O'Brien CA, van Galen P, et al: Variable clonal repopulation dynamics influence chemotherapy response in colorectal cancer. Science 339: 543-548, 2013.

26. Dieter SM, Ball CR, Hoffmann CM, et al: Distinct types of tumor-initiating cells form human colon cancer tumors and metastases. Cell Stem Cell 9: 357-365, 2011. 